\title{
Serological Evidence of Tick-Borne Encephalitis Virus Infection in Rodents Captured at Four Sites in Switzerland
}

\author{
CAROLINE BURRI, ${ }^{1}$ MISA KORVA, ${ }^{2}$ VIKTORIA BASTIC, ${ }^{1}$ NATAŠA KNAP,${ }^{2}$ \\ TATJANA AVŠIČ-ŽUPANC, ${ }^{2}$ AND LISE GERN ${ }^{1,3}$
}

\begin{abstract}
J. Med. Entomol. 49(2): 436-439 (2012); DOI: http://dx.doi.org/10.1603/ME11084
ABSTRACT In a previous study, the presence of tick-borne encephalitis virus (TBEV) in questing Ixodes ricinus $\mathrm{L}$. ticks and in field derived ticks that engorged on small mammals $(n=9,986)$ was investigated at four sites located in a TBE area in Switzerland. Two of these sites were already recognized as TBE foci (Thun and Belp) and the screening of ticks revealed the presence of TBEV in ticks at a third site, Kiesen, but not at the fourth one, Trimstein. The aim here was to test another approach to detect TBE endemic areas. Sera from 333 small mammals (Apodemus flavicollis, A. sylvaticus, Myodes glareolus) captured in 2006 and 2007 at the four sites were examined for the presence of antibodies against TBEV using immunofluorescence and avidity tests. Overall the prevalence of antibodies against TBEV in rodents reached 3.6\% (12/333). At two sites known as TBE foci, Thun and Belp, anti-TBEV antibodies were detected in $9.9 \%(9 / 91)$ and $1.6 \%(1 / 63)$ of rodent sera, respectively. At the third site, Kiesen, recently identified as a TBE focus by the detection of TBEV in ticks, anti-TBEV antibodies were detected in 1.8\% (2/113) of rodent sera. Finally, at Trimstein, none of the examined rodent sera had antibodies against TBEV $(0 / 66)$. This study shows another approach to detect TBE foci by testing antibodies in small mammal sera that is less time-consuming and less expensive than molecular tools.
\end{abstract}

KEY WORDS TBEV, small mammal, antibody, prevalence

Tick-borne encephalitis virus (TBEV) is a flavivirus that accidentally infects human. In Europe, TBEV is transmitted by a tick bite and is maintained in a zoonotic cycle between Ixodes ricinus L. and vertebrate hosts. Ticks may become infected while feeding on a host during the viraemic phase, in so far as level of viraemia is sufficiently high enough (Kunz 1992). As infected hosts rapidly develop specific antibodies and become immune for life (Korenberg 1976) all vertebrates do not act as reservoir hosts (Charrel et al. 2004). However, the viraemic phase is not a necessary condition for TBEV transmission to ticks (Labuda et al. 1993a). Indeed Labuda et al. (1993a) showed a TBEV transmission when ticks were feeding on nonviraemic hosts. This nonviraemic transmission occurs from TBEV infected to uninfected ticks feeding simultaneously and is called cofeeding transmission (Labuda et al. 1993b). Later Labuda et al. (1997) even showed that cofeeding transmission occurs on immune rodent hosts. Thus, small mammals contribute mostly to the TBEV maintenance because they support cofeeding transmission even if they are immune (Labuda et al. 1997) and because of their high repro-

\footnotetext{
${ }^{1}$ Laboratory of Eco-Epidemiology of Parasites, Institute of Biology, University of Neuchâtel, Switzerland.

${ }^{2}$ Institute of Microbiology and Immunology, Faculty of Medicine, University of Ljubljana, Slovenia.

${ }^{3}$ Corresponding author, e-mail: lise.gern@unine.ch.
}

duction rate that decreases the impact of immunization (Shilova 1960 cited in Korenberg 1976).

Previously, we investigated the presence of TBEV in questing $I$. ricinus ticks and in ticks that engorged on rodents $(n=9,986)$ at four sites located in Switzerland (Burri et al. 2011). Among these sites, two of them were known as TBE foci (Thun and Belp), meaning that at least three human cases from each site had been reported to the Federal Office of Public Health. Screening for the presence of the virus in ticks by real-time polymerase chain reaction (RT-PCR) allowed the detection of TBEV at three out of four sites (Thun, Belp, and Kiesen) but not at Trimstein. In the current study, our purpose was to use another approach to detect TBE foci by testing small mammal sera for antibodies against TBEV.

\section{Materials and Methods}

Rodents were trapped once a month during 2006 and 2007 using 50 traps that were set at four sites presenting similar beech grove mesophytic lowland vegetation: Thun $\left(46^{\circ} 43^{\prime} \mathrm{N}, 7^{\circ} 36^{\prime} \mathrm{E}, 642 \mathrm{~m}\right.$ above sea level), Belp ( $46^{\circ} 52^{\prime} \mathrm{N}, 7^{\circ} 30^{\prime} \mathrm{E}, 687 \mathrm{~m}$ above sea level), Kiesen $\left(46^{\circ} 48^{\prime}\right.$ N, $7^{\circ} 34^{\prime}$ E, $566 \mathrm{~m}$ above sea level) and Trimstein $\left(46^{\circ} 53^{\prime} \mathrm{N}, 7^{\circ} 34^{\prime} \mathrm{E}, 620 \mathrm{~m}\right.$ above sea level) (Burri et al. 2011). Rodents were brought to the laboratory. After tick drop-off, rodents were anesthetized intramuscularly with $0.03 \mathrm{ml}$ of Xylasol. (GRAEUB, 
Bern, Switzerland) $(0.02 \mathrm{ml})$ and Ketasol-100 (GRAEUB) $(0.01 \mathrm{ml})$ and blood was obtained from the retro-orbital sinus using Pasteur pipettes $(1.1 \mathrm{~mm}$ diameter). Blood samples were centrifuged twice for $10 \mathrm{~min}$ at $0.8 \mathrm{rcf}$ (relative centrifugal force) and sera were stored at $-20^{\circ} \mathrm{C}$ until processed.

Sera were screened for the presence of TBEV antibodies using a homemade indirect immunofluorescence assay (IFA). TBEV (strain: Ljubljana I, U27494) was inoculated on Vero E6 cell lines and incubated at $37^{\circ} \mathrm{C}$ and $5 \% \mathrm{CO}_{2}$. After $7 \mathrm{~d}$, when characteristic cytopathic effect was noted, cells were centrifuged for $10 \mathrm{~min}$ at $720 \mathrm{rcf}$ and $4^{\circ} \mathrm{C}$. Cells were resuspended in $3 \mathrm{ml} \mathrm{NaCl}$ with $5 \%$ fetal bovine serum (GIBCO, Invitrogen, cat. no. 16415) and $7 \mu \mathrm{l}$ of suspension were put on slides (Biomedicals, cat. no. 096041505), dried, fixed with acetone, and stored at $-20^{\circ} \mathrm{C}$. Before IFA, slides were allowed to dry for $10 \mathrm{~min}$. Seven microliters of rodent sera diluted 1:10 were used for IFA. After 30 min incubation in a moist chamber at room temperature, slides were washed in phosphate buffer saline (PBS; pH 7.4) for $15 \mathrm{~min}$. The antimouse IgG conjugate (A 7506; Sigma, St. Louis, MO) was used at a 1:128 dilution and slides were processed as described above, and examined under a fluorescent microscope (Nikon, eclipse 80i, 400×). Positive and negative controls consisted in one rodent serum that was previously tested positive and PBS ( $\mathrm{pH}$ 7.4), respectively.

Sera from seropositive rodents were further diluted up to 1:2560 to establish the antibody titers and an IFA avidity assay was performed. IFA assay was performed in duplicate. Conditions were the same as described above. After incubation with antimouse IgG conjugate, one set of slides was washed in PBS and the other set with PBS containing $8 \mathrm{M}$ urea. Slides were incubated in PBS with urea for 5 min and then washed with distilled water. The avidity of IgG was calculated as a ratio of the titers obtained from slides washed in PBS and from slides treated with PBS containing urea. An antibody ratio above 32 indicates low IgG avidity and, therefore, an early period of infection (less than $1 \mathrm{mo}$ ) whereas a ratio of four or less indicates high IgG avidity and therefore an infection older than $1 \mathrm{mo}$. After 2 mo of infection, no difference is observed between antibody titers of urea-treated and nontreated specimens.

Sera with antibodies against TBEV were tested for viraemia by amplifying RNA. RNA was isolated using TRIzol (600 $\mu \mathrm{l}$ ) (Invitrogen Life Technologies, Basel, Switzerland) (Chomczynski and Sacchi 1987) and samples were analyzed by RT-PCR (Schwaiger and Cassinotti 2003) as described in Burri et al. (2011). A Fischer's exact test was used for statistical analyses and a $P$ value $\leq 0.05$ was considered as significant.

\section{Results}

Overall, 559 rodents were captured: $278 \mathrm{M}$. glareolus, 161 A. sylvaticus, 118 A. Alavicollis, and 2 Apodemus spp. Trapping was more successful in 2007 (0.2 rodents/trap/night) compared with 2006 (0.06 rodents/ trap/night) (Burri et al. 2011). Details on the number
Table 1. Number of rodents according to species per 100 traps per site and per night in 2006 and 2007

\begin{tabular}{|c|c|c|c|c|c|c|}
\hline \multirow{2}{*}{ Sites } & \multicolumn{2}{|c|}{ A. flavicollis } & \multicolumn{2}{|c|}{ A. sylvaticus } & \multicolumn{2}{|c|}{ M. glareolus } \\
\hline & 2006 & $\overline{2007}$ & 2006 & $\overline{2007}$ & 2006 & 2007 \\
\hline Thun & 0.5 & 3.3 & 3.0 & 5.2 & 3.0 & 8.3 \\
\hline Belp & 0.5 & 2.0 & 0.5 & 6.8 & 1.3 & 7.3 \\
\hline Kiesen & 1.7 & 5.3 & 1.7 & 2.3 & 6.9 & 19.0 \\
\hline Trimstein & 3.1 & 5.5 & 0.6 & 8.8 & 1.4 & 4.0 \\
\hline
\end{tabular}

of collected rodents at each site are shown in Table 1. We analyzed 333 sera from three small mammal species: 152 M. glareolus, 104 A. sylvaticus, and 77 A. flavicollis captured at the four sites in $2006(n=74)$ and $2007(n=259)$. Overall, antibodies against TBEV were detected in $3.6 \%(12 / 333)$ of rodent sera, $6.8 \%$ $(5 / 74)$ in 2006, and $2.7 \%$ (7/259) in 2007 (Fisher exact test; $P>0.05)$. Anti-TBEV antibodies were detected in sera from rodents captured at three sites: Thun, Belp, and Kiesen whereas sera from rodents captured at Trimstein did not show any antibodies (Table 2). The highest prevalence was observed at Thun $(9.9 \%$, 9/91), followed by Kiesen (1.8\%, 2/113), Belp (1.6\%, $1 / 63)$, and Trimstein $(0 \%, 0 / 66)$ (Fisher exact test: Thun/Kiesen, $P=0.01$; Thun/Belp, $P=0.04$; Thun/ Trimstein, $P=0.01$ ) (Table 2 ).

Among the 12 rodents with anti-TBEV antibodies, eight belonged to M. glareolus, three to A. sylvaticus, and one to A. flavicollis (Fisher exact test, $P>0.05$ ) (Table 3). Two M. glareolus (one from Thun, one from Kiesen) had no tick whereas the remaining 10 rodents harbored ticks when they were captured. However, only one A. flavicollis male (Th37) carried TBEV infected ticks (Burri et al. 2011). This individual showed a recent TBEV infection as determined by IgG IFA avidity assay (Table 3 ). Therefore, we tested for viraemia in rodent sera presenting antibodies against TBEV. Screening of the sera by RT-PCR did not reveal the presence of viral RNA in the 12 analyzed serum samples.

\section{Discussion}

This study showed that serological investigation in the rodent population is another approach to detect presence of TBEV in endemic sites. The analysis of only 333 sera from rodents captured in 2006-2007 allowed us to detect the presence of anti-TBEV antibodies at three out of four (Thun, Belp, Kiesen, but not at Trimstein) sites in Switzerland whereas in a previous study, the screening of 9,986 ticks by RT-PCR (Thun: $n=2,001$; Belp: $n=2,117$; Kiesen: $n=4,230$;

Table 2. Prevalence of anti-TBEV antibodies in rodents captured at the four study sites using IFA test

\begin{tabular}{lcl}
\hline \multicolumn{1}{c}{ Sites } & Nb positive/tested sera & $\%$ \\
\hline Thun & $9 / 91$ & 9.9 \\
Belp & $1 / 63$ & 1.6 \\
Kiesen & $2 / 113$ & 1.8 \\
Trimstein & $0 / 66$ & 0 \\
Total & $12 / 333$ & 3.6 \\
\hline
\end{tabular}


Table 3. IFA antibody titers and avidity ratio in rodent sera with anti-TBEV antibodies to predict a recent or a past infection in rodents

\begin{tabular}{|c|c|c|c|c|c|c|c|}
\hline $\begin{array}{c}\text { Sample } \\
\text { ID }\end{array}$ & Species & Sex & End titre & Titre with urea & Avidity ratio & Infection & \\
\hline Thun 9 & M. glareolus & Male & $1: 80$ & $1: 40$ & 2 & High & Old \\
\hline Thun 10 & M. glareolus & Male & $1: 320$ & 1 & 320 & Low & New \\
\hline Thun 12 & M. glareolus & Female & $1: 640$ & $1: 320$ & 2 & High & Old \\
\hline Thun 23 & M. glareolus & Female & $1: 640$ & $1: 40$ & 16 & Equivocal & $?$ \\
\hline Thun 35 & M. glareolus & Male & $1: 40$ & 1 & 40 & Low & New \\
\hline Thun 37 & A. flavicollis & Male & $1: 40$ & 1 & 40 & Low & New \\
\hline Thun 45 & M. glareolus & Male & $1: 80$ & $1: 10$ & 8 & High & Old \\
\hline Thun 51 & A. sylvaticus & Male & $1: 40$ & $1: 40$ & 1 & High & Old \\
\hline Thun 58 & M. glareolus & Male & $1: 640$ & $1: 80$ & 8 & High & Old \\
\hline Kiesen 26 & A. sylvaticus & Female & $1: 80$ & 1 & 80 & Low & New \\
\hline Kiesen 135 & M. glareolus & Female & $1: 80$ & 1 & 80 & Low & New \\
\hline Belp 49 & A. sylvaticus & Female & 1:160 & 1 & 160 & Low & New \\
\hline
\end{tabular}

Trimstein: $n=1,638$ ) was necessary to evaluate the presence/absence of TBEV at the four sites (Burri et al. 2011).

A small proportion of rodents displayed anti-TBEV antibodies, ranging from 1.6 to $9.9 \%$, but these results are consistent with results from Kozuch et al. (1967) and Bakhvalova et al. (2006) who reported antibody prevalence of $4-11 \%$ and $2.6-10.2 \%$, respectively. Interestingly, although more rodents were captured in 2007, seroprevalence in rodent was lower than in 2006. A greater proportion of young individuals in the rodent population could have contributed to dilute the seroprevalence. Unfortunately, we do not have any data on the population structure. Alternatively, at most sites, the low infestation by immature ticks on rodents in 2007 (Burri et al. 2011) may explain the lower prevalence of antibodies in rodents this year.

Thun was the site where the prevalence of antiTBEV antibodies in rodent sera was the highest. In 1977 already, Matile (1982) reported the presence of antibodies against TBEV in four small mammals at this same study site. This observation shows the stability of this focus. At Belp, TBEV in questing I. ricinus ticks (de Marval 1994, Casati et al. 2006, Burri et al. 2011) and anti-TBEV antibodies in rodent sera were detected confirming the presence of the virus at this site. Kiesen and Trimstein were not recognized as TBE foci by the Federal Office of Public Health http://www. bag.admin.ch/themen/medizin/00682/00684/01069/ index.html?lang $=\mathrm{fr}$ ) because no human case has ever been reported at these sites. However, Kiesen should now be considered as a TBE focus because TBEV was recently detected in questing ticks (Burri et al. 2011) and because of the detection of anti-TBEV antibodies in rodents. Trimstein is the only site where TBEV was not detected in ticks (Burri et al. 2011) and where anti-TBEV antibodies were not observed in rodents. Current results do not allow to definitively conclude that TBEV was absent at Trimstein because no significant difference in seroprevalence between any of the sites, except Thun was found. Further studies are needed to confirm the absence of the circulation of TBEV at this site although the results on ticks at this site (Burri et al. 2011) certainly support this conclusion.

No viral RNA was detected in the seropositive samples. It should be noted that the absence of viraemia could be because of the fact that rodents blood was taken after all ticks dropped off (after $7 \mathrm{~d}$ ) whereas viraemia lasts only 2-3 d (Kozuch et al. 1981).

One A. flavicollis that showed a recent TBEV infection by IgG IFA avidity assay was infested by infected larvae $(11.9 \%)$ and uninfected nymphs $(n=5)$ when it was captured (Burri et al. 2011). As Labuda et al. (1993a) showed that A. flavicollis does not develop a viraemia, or does so at a very low level, many alternative explanations for the high prevalence of infection detected in larvae can be given. The presence of TBEV in larvae might be explained by transovarial transmission (Danielová and Holubová 1991). However, the prevalence of TBEV in larvae feeding on this Apodemus mouse was much higher $(11.9 \%)$ (Burri et al. 2011) than the efficiency of transovarial transmission (0.2-0.8\%) described by Danielová and Holubová (1991) suggesting another way of transmission. Therefore cofeeding transmission of TBEV among cofeeding larvae (some of them having been transovarially infected) might be another explanation and as this mode of infection is not absolute (Labuda et al. 1993a), it could explain why the nymphs that were cofeeding with larvae when the animal was captured were uninfected.

The screening of rodent sera for anti-TBEV antibodies revealed to be an appropriate approach to localize the presence of TBEV at sites where TBEV was also detected in ticks (Burri et al. 2011). One advantage of this approach is that it is less time-consuming and less expensive than molecular tools. This IFA avidity assay was not tested against cross reactivity with other flaviviruses such as West Nile virus and Louping-ill virus in Europe. However as West Nile virus is a mosquito borne virus and rodents are not involved in the enzootic cycle and as the Louping-ill virus it is not persistent in small mammals in nature (Gilbert et al. 2000) the risk of cross reactivity is low, at least in the studied sites. Thus, rodents could be used as sentinels to detect TBEV by testing anti-TBEV antibodies in areas suspected to be endemic for TBE. Other animals such as roe deer and dogs were used as sentinels to detect TBEV areas (Gerth et al. 1995, Lindhe et al. 2009). However, small mammals are more appropriate because of their low home range allowing a more precise localization of a TBEV focus. 


\section{Acknowledgments}

We are thankful to Olivier Rais (University of Neuchâtel, Switzerland) for his precious technical assistance. This work is part of the Ph.D. thesis of Caroline Burri and was financed by the Swiss National Science Foundation (FN n $320000-$ 113936).

\section{References Cited}

Bakhvalova, V. N., A. K. Dobrotvorsky, V. V. Panov, V. A. Matveeva, S. E. Tkachev, and O. V. Morozova. 2006. Natural tick-borne encephalitis virus infection among wild small mammals in the southeastern part of western Siberia, Russia. Vector Borne Zoonotic Dis. 6: 32-41.

Burri, C., V. Bastic, G. Maeder, E. Patalas, and L. Gern. 2011. Microclimate and the zoonotic cycle of tick-borne encephalitis virus in Switzerland. J. Med. Entomol. 48: 615627.

Casati, S., L. Gern, and J. C. Piffaretti. 2006. Diversity of the population of tick-borne encephalitis virus infecting $I x$ odes ricinus ticks in an endemic area of central Switzerland (Canton Bern). J. Gen. Virol. 87: 2235-2241.

Charrel, R. N., H. Attoui, A. M. Butenko, J. C. Clegg, V. Deubel, T. V. Frolova, E. A. Gould, T. S. Gritsun, F. X. Heinz, M. Labuda, et al. 2004. Tick-borne virus diseases of human interest in Europe. Clin. Microbiol. Infect. 10: $1040-1055$.

Chomczynski, P., and N. Sacchi. 1987. Single-step method of RNA isolation by acid guanidinium thiocyanate-phenol-chloroform extraction. Anal. Biochem. 162: 156-159.

Danielová, V., and J. Holubová. 1991. Transovarial transmission rate of tick-borne encephalitis virus in Ixodes ricinus ticks, pp. 7-10. In F. Dusbabek and V. Bukva (eds.), Modern acarology (vol. 2). Prague and SPB Academic Publishing, The Hague, The Netherlands.

de Marval, F. 1994. Etude séroépidémiologique de l'encéphalite à tiques et de la borréliose de Lyme en Suisse. Ph.D. dissertation, University of Neuchâtel, Switzerland.

Gerth, H. J., D. Grimshandl, B. Stage, G. Doller, and C. Kunz. 1995. Roe deer as sentinels for endemicity of tick-borne encephalitis virus. Epidemiol. Infect 115: 355-365.

Gilbert, L., L. D. Jones, P. J. Hudson, E. A. Gould, and H. W. Reid. 2000. Role of small mammals in the persistence of
Louping-ill virus: field survey and tick co-feeding studies. Med. Vet. Entomol. 14: 277-282.

Korenberg, E. I. 1976. Some contemporary aspects of natural focality and epidemiology of tick-borne encephalitis. Folia Parasitol. (Praha). 23: 357-366.

Kozuch, O., S. P. Chunikhin, M. Gresíková, J. Nosek, V. B. Kurenkov, and J. Lysý. 1981. Experimental characteristics of viraemia caused by two strains of tick-borne encephalitis virus in small rodents. Acta Virol. 25: 219-224.

Kozuch, O., M. Gresíková, J. Nosek, M. Lichard, and M. Sekeyová. 1967. The role of small rodents and hedgehogs in a natural focus of tick-borne encephalitis. Bull. W.H.O. 36 (Suppl): 61-66.

Kunz, Ch. 1992. Tick-borne encephalitis in Europe. Acta Leidensia 60: 1-14.

Labuda, M., P. A. Nuttall, O. Kozuch, E. Eleckova, T. Williams, E. Zuffova, and A. Sabo. 1993a. Non-viraemic transmission of tick-borne encephalitis virus: a mechanism for arbovirus survival in nature. Experientia 49: 802805.

Labuda, M., L. D. Jones, T. Williams, V. Danielova, and P. A. Nuttall. 1993b. Efficient transmission of tick-borne encephalitis virus between cofeeding ticks. J. Med. Entomol. 30: 295-299.

Labuda, M., O. Kozuch, E. Zuffova, E. Eleckova, R. S. Hails, and P. A. Nuttall. 1997. Tick-borne encephalitis virus transmission between ticks cofeeding on specific immune natural rodent hosts. Virology 235: 138-143.

Lindhe, K. E., D. S. Meldgaard, P. M. Jensen, G. A. Houser, and M. Berendt. 2009. Prevalence of tick-borne encephalitis virus antibodies in dogs from Denmark. Acta Vet. Scand. 51: 56.

Matile, H. 1982. Etudes virologiques et épidémiologiques sur l'encéphalite à tiques en Suisse. Ph.D. dissertation. University of Neuchâtel, Switzerland.

Schwaiger, M., and P. Cassinotti. 2003. Development of a quantitative real-time RT-PCR assay with internal control for the laboratory detection of tick borne encephalitis virus (TBEV) RNA. J. Clin. Virol. 27: 136-145.

Shilova, S. A. 1960. On biological grounds of epidemiological forecast of tick-borne encephalitis. Byull MOIP., otd boil. 65: 37-47.

Received 18 April 2011; accepted 7 December 2011 